\title{
Transcriptional markers of sub-optimal nutrition in developing Apis mellifera nurse workers
}

Vanessa Corby-Harris ${ }^{1,2^{*}}$, Beryl M Jones ${ }^{1,3+}$, Alexander Walton ${ }^{1,4+}$, Melissa R Schwan ${ }^{1}$ and Kirk E Anderson ${ }^{1,2}$

\begin{abstract}
Background: Honey bees (Apis mellifera) contribute substantially to the worldwide economy and ecosystem health as pollinators. Pollen is essential to the bee's diet, providing protein, lipids, and micronutrients. The dramatic shifts in physiology, anatomy, and behavior that accompany normal worker development are highly plastic and recent work demonstrates that development, particularly the transition from nurse to foraging roles, is greatly impacted by diet. However, the role that diet plays in the developmental transition of newly eclosed bees to nurse workers is poorly understood. To further understand honey bee nutrition and the role of diet in nurse development, we used a high-throughput screen of the transcriptome of 3 day and 8 day old worker bees fed either honey and stored pollen (rich diet) or honey alone (poor diet) within the hive. We employed a three factor (age, diet, age $x$ diet) analysis of the transcriptome to determine whether diet affected nurse worker physiology and whether poor diet altered the developmental processes normally associated with aging.
\end{abstract}

Results: Substantial changes in gene expression occurred due to starvation. Diet-induced changes in gene transcription occurring in younger bees were largely a subset of those occurring in older bees, but certain signatures of starvation were only evident 8 day old workers. Of the 18,542 annotated transcripts in the A. mellifera genome, 150 transcripts exhibited differential expression due to poor diet at $3 \mathrm{~d}$ of age compared with 17,226 transcripts that differed due to poor diet at $8 \mathrm{~d}$ of age, and poor diet caused more frequent down-regulation of gene expression in younger bees compared to older bees. In addition, the age-related physiological changes that accompanied early adult development differed due to the diet these young adult bees were fed. More frequent down-regulation of gene expression was observed in developing bees fed a poor diet compared to those fed an adequate diet. Functional analyses also suggest that the physiological and developmental processes occurring in well-fed bees are vastly different than those occurring in pollen deprived bees. Our data support the hypothesis that poor diet causes normal age-related development to go awry.

Conclusion: Poor nutrition has major consequences for the expression of genes underlying the physiology and age-related development of nurse worker bees. More work is certainly needed to fully understand the consequences of starvation and the complex biology of nutrition and development in this system, but the genes identified in the present study provide a starting point for understanding the consequences of poor diet and for mitigating the economic costs of colony starvation.

Keywords: Transcriptome, Apis mellifera, Nutrition, Starvation, Nurse, Development, Physiology

\footnotetext{
*Correspondence: v.corbyharris@gmail.com

${ }^{\dagger}$ Equal contributors

'Carl Hayden Bee Research Center, USDA Agricultural Research Service,

2000 E. Allen Road, Tucson, Arizona 85719, USA

2Department of Entomology, University of Arizona, Tucson, Arizona 85721,

USA

Full list of author information is available at the end of the article
}

\section{Biomed Central}

(C) 2014 Corby-Harris et al.; licensee BioMed Central Ltd. This is an Open Access article distributed under the terms of the Creative Commons Attribution License (http://creativecommons.org/licenses/by/2.0), which permits unrestricted use, distribution, and reproduction in any medium, provided the original work is properly credited. The Creative Commons Public Domain Dedication waiver (http://creativecommons.org/publicdomain/zero/1.0/) applies to the data made available in this article, unless otherwise stated. 


\section{Background}

The European honey bee (Apis mellifera) contributes substantially to the worldwide economy and ecosystem health by pollinating a wide variety of plants [1]. Honey bee colonies function as a unit or superorganism, where facultatively sterile female workers support the queen and her developing brood through a progression of tasks under temporal, genetic, environmental, and physiological control [2,3]. Age is intimately tied to development, and shifts in worker behavior over time are accompanied by dramatic physiological, anatomical, and physiological changes [4-6]. Despite the dramatic nature of these shifts, this network is flexible, where behaviors can accelerate, slow, or revert in response to the changing needs of the colony, such as in times of stress or changes in colony demography $[3,7]$.

Pollen is essential to the diet of larvae and young adult workers [8,9]. Larvae acquire protein through worker jelly, a rich secretion from nurse workers' hypopharyngeal glands (HGs). After eclosion, very young worker bees (less than $\sim 3$ days of age) receive a diet of regurgitated pollen and worker jelly [2]. Between 3 and 8 days of age, workers transition to a diet of beebread, stored pollen mixed with sugars and microbes $[2,10,11]$. Much of the developing adults' protein, lipids, and micronutrients come from beebread, and these nutrients stimulate the growth of HGs and the fat body (reviewed in [10]) in preparation for the physiologically demanding nurse ( 8-14 days) and forager ( 14-21 days) roles. Nurses support developing brood and young adults through their HG secretions, and foragers rely primarily on nutrient stores to supplement the small amount of nutrients consumed via trophallaxis and while foraging for nectar, water, and pollen to support the hive $[10,12]$. Worker pollen consumption increases until nursing age and then subsequently decreases (reviewed in [10]), so the protein assimilation that precedes nursing is critical to colony growth and ontogeny.

Managed honey bees experience long periods of nutritional stress. The majority of honey bees in the U.S. are managed by migratory beekeepers whose livelihood depends on moving hundreds to thousands of colonies to crops requiring pollination. Spring buildup is a crucial time when honey bee colonies that have survived the winter are prepared for pollination of the first crop of the season. Colonies typically come out of winter with little to no pollen stores because any stores from the previous summer and fall were used to sustain the colony through the harsh winter. During spring buildup or throughout the pollination season beekeepers supplement their hives with homemade combinations of protein, sugars, micronutrients, phagostimulants, and antimicrobials that are an incomplete replacement for natural pollen $[10,13]$. Commercially available pollen substitutes are also used and bees consume and colonies grow in response to these substitutes as well as patties containing natural pollen $[14,15]$. Nonetheless, recent surveys of beekeepers in small and large commercial operations rank starvation as a major cause of colony loss $[16,17]$, and so there is still a great need for understanding the basic biology of starvation and for developing markers to assess the sublethal effects of poor diet in young adult honey bees at this critical life stage.

Two recent studies on bees kept in cages away from their hive illustrate the utility of high-throughput methods such as oligonucleotide microarrays and messenger RNA sequencing (mRNA-Seq) for studying the biological signatures of dietary stress in nurse worker honey bees. Using mRNA-Seq in whole abdomens (including the digestive tract), Alaux et al. [18] found that metabolic pathways involved in nutrient-sensing, metabolism, aging, and immunity were up-regulated in nurses fed pollen, while pathways involved in stress response and the regulation of gene expression were down-regulated. Ament et al. [19] used oligonucleotide microarrays to study gene expression in the abdominal carcasses (excluding the digestive tract) of nurses and foragers and found that pollen intake caused the activation of nutrient metabolism processes and reduced expression of transcripts involved in glycolysis, growth and development, neurogenesis, reproduction, and muscle contraction. These two studies used different approaches, yet both find that genes controlling transcriptional regulation and cell signaling are down-regulated in bees fed pollen while those controlling biosynthesis and lipid metabolism are up-regulated $([18,19]$ and see Additional file 1: Table S1, Additional file 2: Table S2 and Additional file 3: Figure S1). However, it is still unclear (1) whether these responses apply to bees in a natural hive setting and (2) how these signatures of incipient starvation accompany the developmental and physiological changes that occur with age. More work must be done to understand how young adult bees respond to poor diet and to understand the basic biology of nutrition in this system.

We aimed to further understand the transcriptional changes associated with starvation during early adult development. To this end, we assayed 3 day old and 8 day old bees kept in normally functioning hives and caged over only honey (poor diet) or honey and bee bread (rich diet) and allowed trophallaxis with the rest of their colony. Whole transcriptomes from abdominal carcasses (i.e., the fat body, the reduced reproductive organs, exoskeleton, trachea, and muscles) were analyzed to focus on changes in the $A$. mellifera abdomen exclusive of the digestive tract. We hypothesized that (1) starvation and aging would greatly impact gene expression and physiology, (2) that starvation would impact young bees differently than older bees, and that (3) early adult development (aging) would be affected by diet. We also aimed 
to provide testable predictions for research on development and nutrition in A. mellifera and develop targets for improving the productivity of bee colonies under nutritional stress. Here, we report that starvation greatly affects the expression of genes related to nurse physiology and development and that the processes that occur as adults develop into nurse bees are altered by sub-optimal diet.

\section{Results}

Bees fed pollen had larger hypopharyngeal glands (HG) compared to those that were not fed pollen

We began by assessing whether bees that were fed only honey (no pollen) had reduced hypopharyngeal glands, a classic signature of starvation. Hypopharyngeal gland (HG) size was measured in twelve classes of bees ( 2 ages $\times$ 2 diets $\times 3$ host colonies). For each of these twelve treatment combinations, HGs were dissected from approximately 5 bees and 10 randomly selected acini were measured for each gland. Hypopharyngeal gland (HG) size was significantly affected by all factors tested in the model $-\operatorname{diet}\left(\mathrm{F}_{1,4}=160.22, \mathrm{p}=0.0002\right)$, host colony (i.e., the host colony that the focal bees were placed into; $\left.\mathrm{F}_{2,8}=13.10, \mathrm{p}=0.0030\right)$, age $\left(\mathrm{F}_{1,4}=39.50, \mathrm{p}=0.0033\right)$, diet by colony $\left(\mathrm{F}_{2,8}=96.80, \mathrm{p}<0.0001\right)$, age by diet $\left(\mathrm{F}_{1,4}=15.97\right.$, $\mathrm{p}=0.0162)$, age by colony $\left(\mathrm{F}_{2,8}=35.89, \mathrm{p}=0.0001\right)$, and the three-way age by diet by colony interaction $\left(\mathrm{F}_{2,4}=13.61\right.$, $\mathrm{p}=0.0164$ ). Post-hoc analyses showed significant differences between $3 \mathrm{~d}$ old bees fed the rich versus poor diet, $8 \mathrm{~d}$ old bees fed the rich versus poor diet, and $3 \mathrm{~d}$ versus $8 \mathrm{~d}$ bees fed the rich diet, but no difference between $3 \mathrm{~d}$ and $8 \mathrm{~d}$ old bees fed only honey (Figure 1).

Due to the significant interaction between diet and colony in the full model discussed above, the effect of diet and age on HG size was also investigated for each colony separately. For colony $\mathrm{A}$, the effect of diet $\left(\mathrm{F}_{1,4}=217.65, \mathrm{p}=0.0001\right)$ and the interaction between age and diet $\left(\mathrm{F}_{1,2}=34.25, \mathrm{p}=0.0280\right)$ were significant but the effect of age $\left(\mathrm{F}_{1,4}=6.80, \mathrm{p}=0.0596\right)$ was not (Additional file 4: Figure S2). For colony $B$, the effect of diet $\left(F_{1,4}=189.38\right.$, $\mathrm{p}=0.0002$ ) and the interaction between age and diet $\left(\mathrm{F}_{1,2}=14.05, \mathrm{p}=0.0200\right)$ were significant but the effect of age $\left(\mathrm{F}_{1,4}=2.85, \mathrm{p}=0.1667\right)$ was not (Additional file 4: Figure S2). For colony $C$, the effect of age $\left(F_{1,4}=72.60\right.$, $\mathrm{p}=0.0010)$ and $\operatorname{diet}\left(\mathrm{F}_{1,4}=26.49, \mathrm{p}=0.0068\right)$ were significant, but the effect of the age $\times$ diet interaction $\left(\mathrm{F}_{1,2}=0.50\right.$, $\mathrm{p}=0.5511$ ) was not (Additional file 4: Figure S2).

The HG sizes of bees that were used to evaluate the predictive power of the mRNA-Seq results with qPCR (but that were not part of the mRNA sequencing experiment), were also measured. Only 8d old bees were used in this phase of the experiment, yielding 6 different classes of bees ( 2 diets $\times 3$ colonies). For each of these 6 different treatment combinations, the HGs of 5 bees were dissected and 10 randomly selected acini were measured

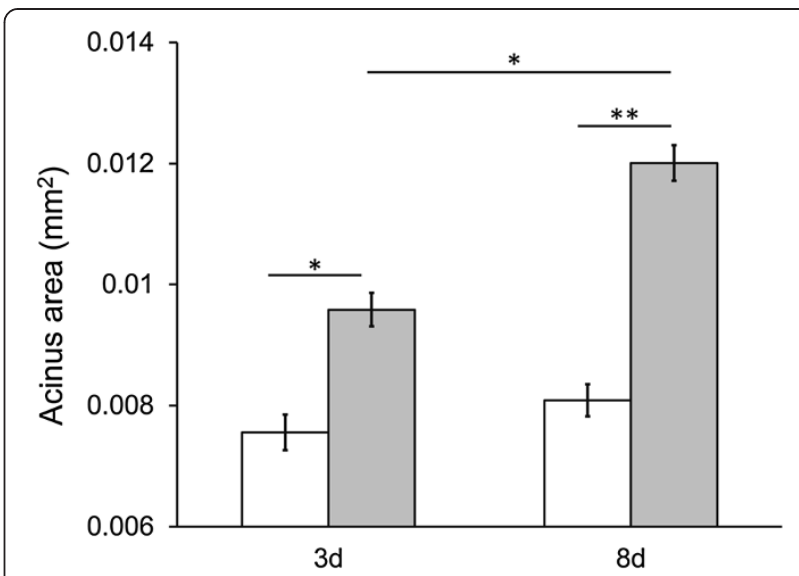

Figure 1 Hypopharyngeal gland (HG) sizes of nurse workers fed or deprived of pollen. $\mathrm{HG}$ size was significantly affected by both diet and age in developing workers fed a diet of pollen and honey (grey bars) or a diet of honey alone (white bars), as measured by HG acinus size $\left(\mathrm{mm}^{2}\right)$. Error bars represent standard error for the mean acinus size across the three colonies $(N=3)$ tested. Asterisks represent the results of a post-hoc Tukey-Kramer test on the mean acinus sizes for each diet by treatment combination and represent either a $p<0.05\left(^{*}\right)$ or $p<0.001\left(^{* *}\right)$ level of significance. There was no significant difference in mean acinus size between $3 \mathrm{~d}$ and $8 \mathrm{~d}$ old bees fed only honey.

for each gland. The HGs from $8 \mathrm{~d}$ old bees fed pollen were significantly larger than those from $8 \mathrm{~d}$ old bees fed only honey ( $8 \mathrm{~d}$ rich diet mean acinus area: $0.015 \mathrm{~mm}^{2} \pm 0.002 \mathrm{~s}$. e., $8 \mathrm{~d}$ poor diet mean acinus area: $0.007 \mathrm{~mm}^{2} \pm 0.0003$ s.e.; $\left.\mathrm{F}_{2,4}=24.48, \mathrm{p}=0.0057\right)$.

\section{Sequencing statistics and broad patterns}

Across all twelve libraries ( 2 ages $\times 2$ diets $\times 3$ host colonies), approximately 149.4 million paired end reads passed the initial quality control filters and approximately 103 million paired end reads mapped to the $A$. mellifera genome (Additional file 5: Table S3). Across the twelve libraries, an average of 8.58 million (67.5\%) paired end reads mapped to the $A$. mellifera genome per library. In total, the expression of 67,002 exons (non-singleton exons; see Methods) mapping to 12,340 transcripts (containing $\geq 2$ exons; see Methods) was differentially expressed (Additional file 6: Table S4 [20]). The interaction between diet and age did not significantly impact the expression of any exons, and so results are presented below for exons and transcripts that changed due to the main effect of diet, the main effect of age, the effect of diet at either $3 \mathrm{~d}$ or $8 \mathrm{~d}$, or the effect of age in bees fed a rich or poor diet.

Starvation caused drastic differences in gene expression, and these differences are most evident when the effect of starvation is assessed for each age class separately

We investigated whether starvation impacted gene expression by first testing whether transcription was affected by 
the main effect of diet (i.e., exhibited consistent differences due to diet in both $3 \mathrm{~d}$ and $8 \mathrm{~d}$ bees). The expression of 24 exons mapping to 13 transcripts was significantly affected by the main effect of diet and all were up-regulated in bees fed pollen compared to those that were fed a poor diet (Table 1). These 24 up-regulated exons, which included transcripts encoding vitellogenin and worker-enriched antennal transcript, mapped to 5 orthologues (Additional file 2: Table S2 [20]) but were not associated with any biological processes or gene annotation clusters.

The genes that were up- or down-regulated due to starvation in either young ( $3 \mathrm{~d}$ old) or old ( $8 \mathrm{~d}$ old) bees were analyzed separately to determine whether the effects of starvation varied with the age of the bee. Starvation caused more frequent down-regulation of transcripts in younger bees (150 out of $173 ; 78.5 \%$ ) compared to older bees $\left(11,393\right.$ out of 20,$376 ; 55.9 \% ; X_{1}^{2}=64.82, p<0.0001$; Table 2 and Additional file 6: Table S4 [20]). Comparisons using exons instead of genes containing $\geq 2$ exons per transcript yielded similar results $\left(X_{1}^{2}=144.83, p<0.0001\right.$; Additional file 7: Table S5).

We determined how patterns of diet-induced gene upor down-regulation overlapped between the two age classes. Transcripts and orthologues that changed with diet at $3 \mathrm{~d}$ of age were largely a subset of those that changed at $8 \mathrm{~d}$ of age (Figure 2). Transcripts down-regulated due to starvation in both $3 \mathrm{~d}$ and $8 \mathrm{~d}$ old bees (Figure 2, left side, rich $>$ poor) included those encoding cuticular proteins, apidermins, worker-enriched antennal transcript, and nitric oxide synthase (Additional file 6: Table S4 [20]) and were associated with chitin metabolism, response to oxidative stress, and motor function (Table 3). Transcripts up-regulated in pollen deprived bees at both time points (Figure 2, right side, poor $>$ rich) included those encoding a histone-arginine methyltransferase, dynein heavy chain, dual oxidase (Duox), and argonaute (Additional file 6:
Table S4 [20]) but were not significantly related to any biological processes or gene annotation clusters.

The genes were down-regulated upon starvation exclusively at $3 \mathrm{~d}$ or $8 \mathrm{~d}$ of age (Figure 2 , left side, rich $>$ poor) were assessed to determine whether the processes associated with starvation differed in young versus old bees. Transcripts down-regulated due to starvation in young (3d old) but not old ( $8 \mathrm{~d}$ old) bees included apidermin 3 , cuticular proteins, and ecdysis triggering hormone (Additional file 6: Table S4 [20]) but no biological processes or annotation clusters. Transcripts down-regulated in pollen deprived bees at $8 \mathrm{~d}$ but not at $3 \mathrm{~d}$ included glutathione $\mathrm{S}$-transferases, major royal jelly (MRJP) proteins 1 through 9, hexamerins, DNA methyltransferases, cytochrome p450s, and immune genes (Additional file 6: Tables S4 [20]) but were not associated with any biological processes or gene annotation clusters.

Genes that were up-regulated upon starvation exclusively at $3 \mathrm{~d}$ or $8 \mathrm{~d}$ of age were next assessed (Figure 2, right side, poor $>$ rich) to determine whether the increases in gene expression that occurred differed in young and old bees. One transcript encoding transient receptor potential-gamma protein-like (XM_001122469) was up-regulated in pollen deprived bees exclusively at 3d (Additional file 6: Tables S4 [20]) but this transcript was not orthologous to a D. melanogaster gene. Transcripts up-regulated in pollen deprived bees exclusively at $8 \mathrm{~d}$ included immune recognition genes, cytochrome P450s, cuticular proteins, MRJPs, hexamerins, DNA methyltransferases, and one microRNA (Additional file 6: Tables S4 [20]) but were not associated with any biological processes or annotation clusters.

\section{Starvation alters normal age-associated nurse development}

To ask whether certain signatures of early adult development were consistently expressed in the same direction

Table 1 Differentially expressed exons, transcripts, and orthologues for each category investigated

\begin{tabular}{|c|c|c|c|c|c|c|c|c|c|c|c|c|c|}
\hline & \multicolumn{6}{|c|}{ Diet } & \multicolumn{6}{|c|}{ Age } & \multirow[t]{3}{*}{ Average } \\
\hline & \multicolumn{3}{|c|}{$\uparrow$ Rich } & \multicolumn{3}{|c|}{$\uparrow$ Poor } & \multicolumn{3}{|c|}{$\uparrow$ Young } & \multicolumn{3}{|c|}{$\uparrow$ Old } & \\
\hline & Main & $3 d$ & $8 d$ & Main & $3 d$ & $8 d$ & Main & Rich diet & Poor diet & Main & Rich diet & Poor diet & \\
\hline Exons $^{A}$ & 24 & 360 & 42,497 & 0 & 26 & 24,314 & 187 & 91 & 184 & 76 & 94 & 72 & \\
\hline Transcripts $^{B}$ & 13 & 150 & 11,393 & 0 & 23 & 8,983 & 81 & 39 & 77 & 18 & 23 & 14 & \\
\hline Annotated transcripts ${ }^{c}$ & 11 & 134 & 9,642 & 0 & 16 & 7,584 & 74 & 33 & 71 & 18 & 21 & 13 & \\
\hline $\begin{array}{l}\text { A. mellifera transcripts } \\
\text { placed with } D \text {. melanogaster } \\
\text { orthologues }^{D}\end{array}$ & 5 & 59 & 4,580 & - & 6 & 3,569 & 48 & 9 & 49 & 4 & 8 & 3 & \\
\hline $\begin{array}{l}\text { Percent of total transcripts } \\
\text { identified in the present } \\
\text { study with an orthologue }\end{array}$ & 38 & 39 & 40 & - & 26 & 40 & 59 & 23 & 64 & 22 & 35 & 21 & 37 \\
\hline
\end{tabular}

ADoes not contain singletons (see Methods).

${ }^{B}$ Differentially expressed transcripts containing $\geq 2$ exons

${ }^{C}$ Differentially expressed transcripts annotated in GenBank. These values correspond to those presented in Figures $2 \mathrm{a}$ and $3 a$.

DThese values correspond to those presented in Figures $2 \mathrm{~b}$ and $3 \mathrm{~b}$.

ETotal number of transcripts includes both the annotated and unannotated transcripts. 
Table 2 Transcript-based analysis of expression with longer periods of starvation or increasing age

\begin{tabular}{|c|c|c|c|c|c|c|c|}
\hline \multirow[t]{2}{*}{ Factor } & \multirow[t]{2}{*}{ Constant } & \multirow{2}{*}{$\begin{array}{l}\text { Transcripts } \\
\text { changed }^{A}\end{array}$} & \multirow[t]{2}{*}{ Increased $^{A}$} & \multirow[t]{2}{*}{ Decreased $^{A}$} & \multirow{2}{*}{$\begin{array}{c}\text { \% Transcripts } \\
\text { decreased }^{\mathrm{B}}\end{array}$} & \multicolumn{2}{|c|}{ Difference in \% decrease? } \\
\hline & & & & & & $x^{2}$ & $p$ \\
\hline \multirow[t]{2}{*}{ Starvation } & $3 d$ & 173 & 23 & 150 & 78.5 & 64.82 & $<0.0001$ \\
\hline & $8 d$ & 20,376 & 8,983 & 11,393 & 55.9 & & \\
\hline \multirow[t]{2}{*}{ Aging } & Poor diet & 91 & 14 & 77 & 79.3 & 8.33 & 0.0039 \\
\hline & Rich diet & 62 & 23 & 39 & 62.9 & & \\
\hline
\end{tabular}

${ }^{\mathrm{A}}$ Differentially expressed transcripts contain $\geq 2$ exons. Transcripts containing single exons were omitted from the analysis.

${ }^{B}$ Of the transcripts that differed due to each factor combination.

and in similar magnitude for well-fed and underfed workers, we determined whether the main effect of age significantly impacted gene expression. A total of 263 exons (mapping to 99 transcripts) were differentially expressed due to the main effect of age (Table 1). 187 (71\%) of the exons (mapping to 81 transcripts) were down-regulated and 76 (29\%) of the exons (mapping to 18 transcripts) were up-regulated as bees as they aged from $3 \mathrm{~d}$ to $8 \mathrm{~d}$ old (Table 1). The transcripts downregulated with age included those coding for vitellogenin, tetraspanin 6, and AncR-1 non-coding nuclear RNA (Additional file 6: Table S4 [20]) and corresponded to 48 orthologues associated with transcriptional regulation (Table 4). The transcripts up-regulated with age included hexamerins, immune genes, juvenile hormone esterase, and odorant binding proteins (Additional file 6: Table S4 [20]) but were not associated with any biological processes or annotation clusters.

Transcripts that were up- or down-regulated with age were investigated in pollen deprived and well-fed bees separately to determine whether the signatures of normal age-associated development differed in underfed versus well-fed bees. Transcript expression decreased with age more frequently in bees fed a poor diet (77 out of $91 ; 79.3 \%)$ compared to bees fed a rich diet (39 out of 62; 62.9\%; $X_{1}^{2}=8.33, p=0.0039$; Table 2 and Additional file 6: Table S4 [20]). Exon-based analyses also supported this trend: $71.9 \%$ of exons were down-regulated with age
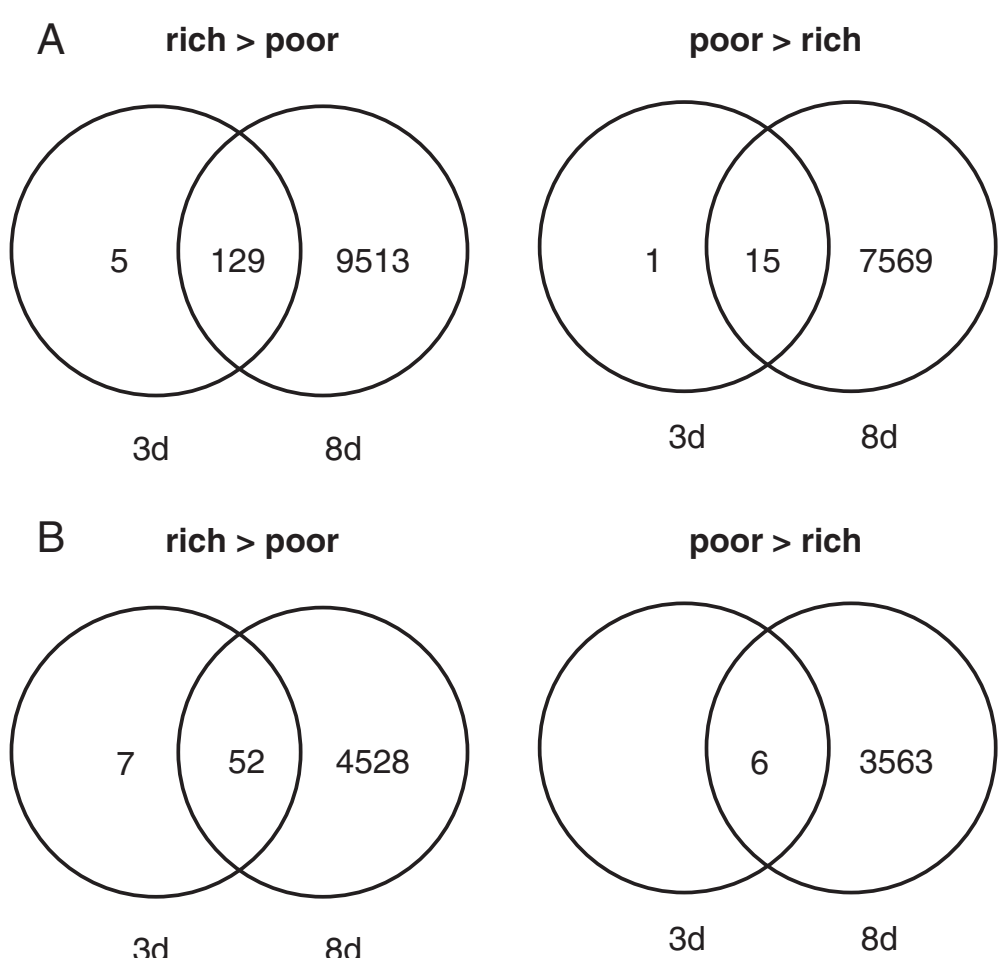

Figure 2 The impact of diet on bees aged $\mathbf{3}$ days or $\mathbf{8}$ days. The number of (A) annotated transcripts and (B) orthologues differentially expressed as a function of diet in bees fed a rich or poor diet for $3 \mathrm{~d}$ or $8 \mathrm{~d}$. Panels on the left (rich > poor) represent terms down-regulated with starvation. Panels on the right (poor > rich) represent terms whose expression was higher in bees fed a poor diet. 
Table 3 Biological process (BP) terms and annotation clusters down-regulated in pollen deprived bees at $\mathbf{3 d}$ and $8 \mathrm{~d}$

\begin{tabular}{|c|c|c|c|c|}
\hline BP term or cluster & Description & $\mathbf{N}$ & $X_{\mathrm{adj}}^{2 \mathrm{~A}}$ & Orthologues $^{\mathrm{B}}$ \\
\hline GO:0007155 & Cell adhesion $(\mathrm{BP})^{\mathrm{C}}$ & 6 & $<0.0001$ & $\begin{array}{l}\text { FBgn0034660, FBgn0000547, } \\
\text { FBgn0031879, FBgn0000464, } \\
\text { FBgn0000117, FBgn0026562 }\end{array}$ \\
\hline GO:0007155, GO:0008360 & $\begin{array}{l}\text { Cell adhesion }(B P)^{C} \text {, regulation } \\
\text { of cell shape }(B P)^{C}\end{array}$ & 3 & $<0.0001$ & $\begin{array}{l}\text { FBgn0000547, FBgn0000464, } \\
\text { FBgn0000117 }\end{array}$ \\
\hline GO:0007391 & Dorsal closure $(\mathrm{BP})^{\mathrm{C}}$ & 3 & 0.0037 & $\begin{array}{l}\text { FBgn0000299, FBgn0000547, } \\
\text { FBgn0000117 }\end{array}$ \\
\hline GO:0046331 & Lateral inhibition $(\mathrm{BP})^{\mathrm{C}}$ & 4 & 0.0059 & $\begin{array}{l}\text { FBgn0010473, FBgn0000547, } \\
\text { FBgn0243514, FBgn0053196 }\end{array}$ \\
\hline IPR002172 & $\begin{array}{l}\text { Low-density lipoprotein (LDL) } \\
\text { receptor class A repeat }\end{array}$ & 3 & $<0.0001$ & $\begin{array}{l}\text { FBgn0031879, FBgn0053196, } \\
\text { FBgn0261341 }\end{array}$ \\
\hline IPR013032 & EGF-like region, conserved site & 5 & $<0.0001$ & $\begin{array}{l}\text { FBgn0003137, FBgn0026403, } \\
\text { FBgn0031879, FBgn0243514, } \\
\text { FBgn0053196 }\end{array}$ \\
\hline GO:0005509 & Calcium ion binding (MF) & 7 & $<0.0001$ & $\begin{array}{l}\text { FBgn0026403, FBgn0031879, } \\
\text { FBgn0000079, FBgn0031464, } \\
\text { FBgn0037963, FBgn0053196, } \\
\text { FBgn0026562 }\end{array}$ \\
\hline IPR003599, IPR007110 & $\begin{array}{l}\text { Immunoglobulin subtype, } \\
\text { Immunoglobulin-like } 6\end{array}$ & 6 & $<0.0001$ & $\begin{array}{l}\text { FBgn0003137, FBgn0010473, } \\
\text { FBgn0000547, FBgn0005666, } \\
\text { FBgn0000464, FBgn0004369 }\end{array}$ \\
\hline $\begin{array}{l}\text { IPR003599, IPR007110, } \\
\text { IPR003961, IPR008957 }\end{array}$ & $\begin{array}{l}\text { Immunoglobulin subtype, Immunoglobulin-like, } \\
\text { Fibronectin, type III, Fibronectin type III domain }\end{array}$ & 5 & $<0.0001$ & $\begin{array}{l}\text { FBgn0010473, FBgn0000547, } \\
\text { FBgn0005666, FBgn0000464, } \\
\text { FBgn0004369 }\end{array}$ \\
\hline $\begin{array}{l}\text { IPR001660, IPR010993, } \\
\text { IPR011510, IPR021129 }\end{array}$ & $\begin{array}{l}\text { Sterile alpha motif domain, Sterile alpha } \\
\text { motif homology, Sterile alpha motif, type 2, } \\
\text { Sterile alpha motif, type } 1\end{array}$ & 3 & $<0.0001$ & $\begin{array}{l}\text { FBgn0000182, FBgn0031762, } \\
\text { FBgn0039075 }\end{array}$ \\
\hline IPR018247 & EF-Hand 1, calcium-binding site & 3 & 0.0019 & $\begin{array}{l}\text { FBgn0031464, FBgn0013809, } \\
\text { FBgn0026562 }\end{array}$ \\
\hline GO:0004867 & $\begin{array}{l}\text { Serine-type endopeptidase } \\
\text { inhibitor activity (MF) }\end{array}$ & 3 & $<0.0001$ & $\begin{array}{l}\text { FBgn0003137, FBgn0026721, } \\
\text { FBgn0053196 }\end{array}$ \\
\hline $\begin{array}{l}\text { GO:0006030, GO:0008061, } \\
\text { IPR002557 }\end{array}$ & $\begin{array}{l}\text { Chitin metabolic process }(B P)^{C} \text {, chitin } \\
\text { binding (MF), Chitin binding domain }\end{array}$ & 3 & $<0.0001$ & $\begin{array}{l}\text { FBgn0027600, FBgn0051973, } \\
\text { FBgn0261341 }\end{array}$ \\
\hline IPR017853 & Glycoside hydrolase, superfamily & 3 & $<0.0001$ & $\begin{array}{l}\text { FBgn0053080, FBgn0000079, } \\
\text { FBgn0026415 }\end{array}$ \\
\hline GO:0030036 & Actin cytoskeleton organization ${ }^{\mathrm{D}}$ & 2 & 0.0080 & FBgn0000182, FBgn0085447 \\
\hline GO:0006470 & Protein dephosphorylation ${ }^{\mathrm{D}}$ & 2 & 0.0242 & FBgn0000464, FBgn0004369 \\
\hline GO:0007498 & Mesoderm development ${ }^{\mathrm{D}}$ & 2 & 0.0130 & FBgn0005666, FBgn0026562 \\
\hline GO:0008045 & Motor axon guidance ${ }^{D}$ & 2 & 0.0013 & FBgn0000464, FBgn0004369 \\
\hline GO:0005975 & Carbohydrate metabolic process ${ }^{\mathrm{D}}$ & 2 & 0.0008 & FBgn0053080, FBgn0000079 \\
\hline GO:0007414 & Axonal defasciculation ${ }^{\mathrm{D}}$ & 2 & $<0.0001$ & FBgn0010473, FBgn0004369 \\
\hline GO:0007156 & Homophilic cell adhesion ${ }^{\mathrm{D}}$ & 2 & 0.0001 & FBgn0000547, FBgn0037963 \\
\hline GO:0006979 & Response to oxidative stress ${ }^{\mathrm{D}}$ & 2 & 0.0033 & FBgn0031464, FBgn0036756 \\
\hline GO:0016337 & Cell-cell adhesion ${ }^{\mathrm{D}}$ & 2 & $<0.0001$ & FBgn0000547, FBgn0000117 \\
\hline
\end{tabular}

${ }^{\mathrm{A}} X_{\mathrm{adj}}^{2}$ refers to the significance of the Fisher's exact test with a Benjamini-Hochberg correction for multiple comparisons.

${ }^{B}$ Orthologues correspond to those pictured in Figure 2B (left panel) and represent Drosophila melanogaster genes that are listed in FlyBase.

${ }^{C}$ Terms that were significant BP terms and were part of a significant gene annotation cluster.

DTerms that represented significant BP terms but did not form a significant gene annotation cluster.

in pollen deprived bees compared to the $49.2 \%$ downregulated with age in bees fed pollen $\left(X_{1}^{2}=22.59, p<0.0001\right.$; Additional file 7: Table S5).

The overlap between transcripts differentially regulated due to age in bees fed either diet was investigated to determine what processes that were up- or downregulated with age were common between underfed and well-fed bees. Only one transcript (with no orthologue), encoding a hypothetical protein (Additional file 6: Table S4 [20]), was down-regulated with age in bees fed both 


\begin{tabular}{|c|c|c|c|c|}
\hline Cluster & Description & $\mathrm{N}$ & $X_{\mathrm{adj}}^{2} \mathrm{~A}$ & Orthologues $^{B}$ \\
\hline GO:0005524 & ATP binding (MF) & 10 & 0.0029 & $\begin{array}{l}\text { FBgn0260990, FBgn0261014, } \\
\text { FBgn0032243, FBgn0025743, } \\
\text { FBgn0026059, FBgn0000723, } \\
\text { FBgn0030343, FBgn0015615, } \\
\text { FBgn0036486, FBgn0051729 }\end{array}$ \\
\hline Kegg:03040 & Spliceosome & 3 & 0.0099 & $\begin{array}{l}\text { FBgn0031390, FBgn0038927, } \\
\text { FBgn0031266 }\end{array}$ \\
\hline GO:0007391 & Dorsal closure $(\mathrm{BP})^{\mathrm{C}}$ & 3 & 0.0048 & $\begin{array}{l}\text { FBgn0010434, FBgn0000723, } \\
\text { FBgn0044323 }\end{array}$ \\
\hline $\begin{array}{l}\text { IPR001680, IPR011046, } \\
\text { IPR019775 }\end{array}$ & $\begin{array}{l}\text { WD40 repeat, WD40 repeat-like-containing } \\
\text { domain, WD40 repeat, conserved site }\end{array}$ & 5 & $<0.0001$ & $\begin{array}{l}\text { FBgn0037758, FBgn0029903, } \\
\text { FBgn0044323, FBgn0038927, } \\
\text { FBgn0043362 }\end{array}$ \\
\hline $\begin{array}{l}\text { IPR001680, IPR011046, } \\
\text { IPR019775, IPR020472, } \\
\text { IPR019782, IPR017986, } \\
\text { IPR019781 }\end{array}$ & $\begin{array}{l}\text { WD40 repeat, WD40 repeat-like-containing } \\
\text { domain, WD40 repeat, conserved site, G-protein } \\
\text { beta WD-40 repeat, WD40 repeat 2, WD40-repeat- } \\
\text { containing domain, WD40 repeat, subgroup }\end{array}$ & 4 & $<0.0001$ & $\begin{array}{l}\text { FBgn0029903, FBgn0044323, } \\
\text { FBgn0038927, FBgn0043362 }\end{array}$ \\
\hline IPR011046 & WD40 repeat-like-containing domain & 6 & $<0.0001$ & $\begin{array}{l}\text { FBgn0037758, FBgn0029903, } \\
\text { FBgn0024698, FBgn0044323, } \\
\text { FBgn0038927, FBgn0043362 }\end{array}$ \\
\hline GO:0003779 & Actin binding (MF) & 3 & 0.0109 & $\begin{array}{l}\text { FBgn0035347, FBgn0010434, } \\
\text { FBgn0029903 }\end{array}$ \\
\hline IPR016024 & Armadillo-type fold & 4 & 0.0248 & $\begin{array}{l}\text { FBgn0260990, FBgn0030674, } \\
\text { FBgn0043362, FBgn0031266 }\end{array}$ \\
\hline GO:0016319 & Mushroom body development ${ }^{\mathrm{D}}$ & 2 & 0.0071 & FBgn0025743, FBgn0010051 \\
\hline GO:0007030 & Golgi organization ${ }^{\mathrm{D}}$ & 2 & 0.0019 & FBgn0261014, FBgn0033075 \\
\hline
\end{tabular}

${ }^{\mathrm{A}} X_{\mathrm{adj}}^{2}$ refers to the significance of the Fisher's exact test with a Benjamini-Hochberg correction for multiple comparisons.

${ }^{\mathrm{B}}$ Orthologues represent Drosophila melanogaster genes similar to the differentially expressed transcripts discovered in the present study that are listed in FlyBase.

${ }^{C}$ Terms that were significant BP terms and were part of a significant gene annotation cluster.

DTerms that represented significant BP terms but did not form a significant gene annotation cluster.

diets. Transcripts up-regulated with age in bees fed either diet included apidaecin, cytochrome P450 342A1, and hexamerin 110 (Additional file 6: Table S4 [20]). These transcripts were associated with one orthologue that was not associated with a biological process or annotation cluster.

The transcripts down-regulated with age in either pollen deprived or well-fed bees were investigated (Figure 3, left panels, young>old) to determine how ageassociated decreases in gene expression differed with diet. Transcripts down-regulated in older bees fed only the rich diet included apidermin and worker anntenal transcript (Additional file 6: Table S4 [20]) and these transcripts were involved in open tracheal system development and chitin metabolism (Table 5). Transcripts down-regulated with age in bees fed only the poor diet included AncR-1 non-coding nuclear RNA (Ancr-1) and tetraspanin 6 (Additional file 6: Table S4 [20]) and were involved in transcription, development, sensory system development, and mRNA splicing (Table 6).

Transcripts up-regulated with age in either pollen deprived or well-fed bees were investigated (Figure 3, right panels, young<old) to determine how age-associated increases in expression differed with diet. Transcripts up- regulated in older bees fed only the rich diet included those encoding apolipophorin and peptidoglycan recognition proteins S2 and S3 (Additional file 6: Table S4 [20]) and were associated with immunity and oxidationreduction (Table 7). Transcripts up-regulated in older bees fed only the poor diet included juvenile hormone esterase, vitellogenin, hexamerin 70a, cytochrome P450 4AZ1 and two odorant binding proteins (Additional file 6: Table S4 [20]). The two orthologues related to these transcripts were not associated with any biological processes or annotation clusters.

\section{Targeted rt-PCR of differentially expressed genes confirmed the mRNA-sequencing results}

To verify our results from the mRNA-seq experiments, real-time PCR was used on a small subset of the genes that were differentially regulated according to the mRNA-seq analyses. The expression of 6 transcripts in the abdominal carcass of $8 \mathrm{~d}$ old bees from three colonies fed honey or honey and bee bread confirmed the mRNA-seq results. The expression of vermiform, vitellogenin, and GTP-binding protein 10 in bees fed a rich diet compared to those fed a poor diet were all greater than one $(\mathrm{p}=0.049$ for each), and the expression of 

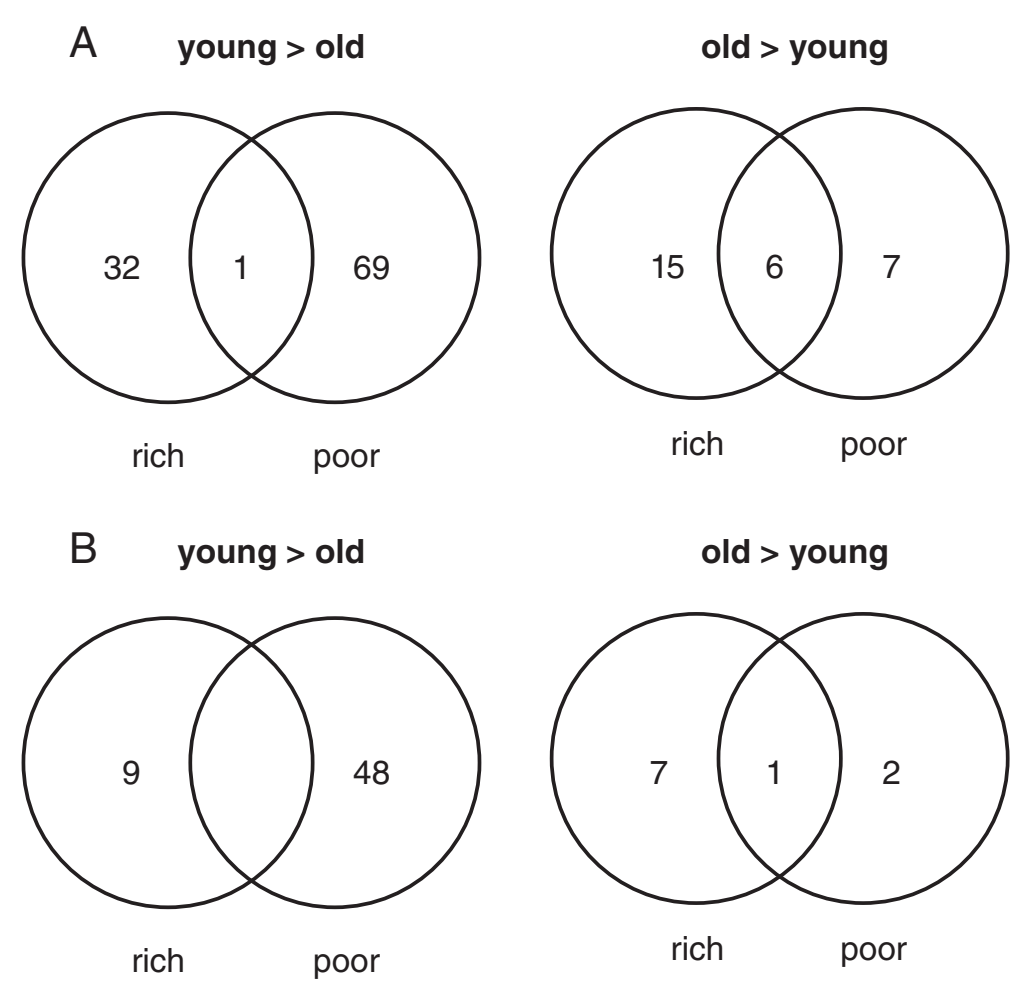

Figure 3 The impact of increasing age for bees fed a rich or poor diet. The number of (A) annotated transcripts and (B) orthologues differentially expressed as a function of age in bees fed a rich or poor diet. Panels on the left (young $>$ old) represent terms down-regulated with age in bees fed either a rich or poor diet. Panels on the right (old > young) represent terms whose expression was up-regulated with age in bees fed either a rich or poor diet.

glucocerebrosidase was significantly lower $(\mathrm{p}=0.049$; Figure 4). The expression of Cdc42 and E75 trended upward $(>1)$ in bees fed a rich diet compared to those fed a poor diet, but their relative expression was not significantly different than one (Figure 4). In each case, the direction of expression change agreed with the predictions from the mRNA-sequencing. All rt-PCR results using the GAPDH control were similar to those using the actin control. In sum, the rt-PCR results verified the mRNA-seq results for the small set of transcripts that were tested.

\section{Discussion}

Pollen is a critical component of the diet for young adult bees and can greatly impact their development. Surveys of beekeepers suggest that starvation is a major cause of colony losses and our understanding of how starvation affects bees at the critical young adult stage is incomplete. To understand the basic biology of pollen deprivation and to develop transcriptional markers of starvation in developing nurse worker bees, we used mRNA-seq to measure changes in gene expression in $3 \mathrm{~d}$ and $8 \mathrm{~d}$ old bees fed a diet of honey and stored pollen within the hive compared to a restricted diet containing honey but no pollen. Many interesting results became apparent when diet-related transcripts were studied separately for bees aged $3 \mathrm{~d}$ or $8 \mathrm{~d}$ and by determining which transcripts were differentially expressed at either $3 \mathrm{~d}$ or $8 \mathrm{~d}$ of age. Substantial changes in gene expression were observed when bees were deprived of pollen and these changes were more numerous as bees were deprived of pollen for longer periods of time. Additionally, normal patterns of gene expression that accompanied agerelated changes in development were altered in underfed

Table 5 Biological process (BP) terms down-regulated with age only in bees fed the rich diet

\begin{tabular}{lllll}
\hline BP term & Description & N & $\boldsymbol{X}_{\text {adj }}^{\mathbf{A}}$ & Orthologues $^{\mathbf{B}}$ \\
\hline GO:0007424 & Open tracheal system development & 2 & $<0.0001$ & FBgn0260653, FBgn0261341 \\
GO:0006030 & Chitin metabolic process & 3 & $<0.0001$ & FBgn0051973, FBgn0260653, FBgn0261341 \\
GO:0035159 & Regulation of tube length, open tracheal system & 2 & $<0.0001$ & FBgn0260653, FBgn0261341 \\
\hline
\end{tabular}

${ }^{\mathrm{A}} X_{\mathrm{adj}}^{2}$ refers to the significance of the Fisher's exact test with a Benjamini-Hochberg correction for multiple comparisons.

${ }^{B}$ Orthologues correspond to those pictured in Figure 3B (left panel) and represent Drosophila melanogaster genes that are listed in FlyBase. 
Table 6 Biological process (BP) terms and annotation clusters down-regulated with age only in pollen deprived bees

\begin{tabular}{|c|c|c|c|c|}
\hline BP term or cluster & Description & $\mathrm{N}$ & $X_{\mathrm{adj}}^{2 \mathrm{~A}}$ & Orthologues $^{B}$ \\
\hline GO:0007391 & Dorsal closure $(\mathrm{BP})^{\mathrm{C}}$ & 3 & $<0.0001$ & FBgn0044323, FBgn0010434, FBgn0000723 \\
\hline GO:0005488 & Binding (MF) & 3 & 0.0357 & FBgn0031266, FBgn0021847, FBgn0030674 \\
\hline GO:0046331 & Lateral inhibition $(\mathrm{BP})^{\mathrm{C}}$ & 3 & 0.0075 & FBgn0029685, FBgn0015615, FBgn0025743 \\
\hline IPR016024 & Armadillo-type fold & 4 & $<0.0001$ & $\begin{array}{l}\text { FBgn0031266, FBgn0043362, FBgn0260990, } \\
\text { FBgn0030674 }\end{array}$ \\
\hline GO:0005524 & ATP binding (MF) & 9 & $<0.0001$ & $\begin{array}{l}\text { FBgn0051729, FBgn0036486, FBgn0261014, } \\
\text { FBgn0032243, FBgn0015615, FBgn0025743, } \\
\text { FBgn0026059, FBgn0260990, FBgn0000723 }\end{array}$ \\
\hline $\begin{array}{l}\text { GO:0005524, GO:0006468, } \\
\text { IPR002290, IPR000719 }\end{array}$ & $\begin{array}{l}\text { ATP binding (MF),protein phosphorylation } \\
\text { (BP), Serine/threonine-protein kinase domain, } \\
\text { Protein kinase, catalytic domain }\end{array}$ & 3 & 0.0080 & FBgn0025743, FBgn0260990, FBgn0000723 \\
\hline IPR011046 & WD40 repeat-like-containing domain & 7 & $<0.0001$ & $\begin{array}{l}\text { FBgn0024698, FBgn0044323, FBgn0027518, } \\
\text { FBgn0043362, FBgn0037758, FBgn0029903, } \\
\text { FBgn0038927 }\end{array}$ \\
\hline GO:0003779 & Actin binding (MF) & 3 & $<0.0001$ & FBgn0010434, FBgn0029903, FBgn0035347 \\
\hline GO:0000398 & Nuclear mRNA splicing, via spliceosome $(B P)^{C}$ & 3 & 0.0080 & FBgn0031266, FBgn0034002, FBgn0038927 \\
\hline Kegg:03040 & Spliceosome & 3 & $<0.0001$ & FBgn0031266, FBgn0038927, FBgn0031390 \\
\hline GO:0006468 & Protein phosphorylation ${ }^{\mathrm{D}}$ & 3 & 0.0148 & FBgn0025743, FBgn0260990, FBgn0000723 \\
\hline GO:0006378 & mRNA polyadenylation ${ }^{\mathrm{D}}$ & 2 & $<0.0001$ & FBgn0024698, FBgn0260780 \\
\hline GO:0016319 & Mushroom body development ${ }^{\mathrm{D}}$ & 2 & $<0.0001$ & FBgn0010051, FBgn0025743 \\
\hline GO:0048749 & Compound eye development ${ }^{\mathrm{D}}$ & 2 & 0.00488 & FBgn0043362, FBgn0025743 \\
\hline GO:0006813 & Potassium ion transport ${ }^{\mathrm{D}}$ & 2 & $<0.0001$ & FBgn0037244, FBgn0037758 \\
\hline GO:0006281 & DNA repair ${ }^{\mathrm{D}}$ & 2 & $<0.0001$ & FBgn0036486, FBgn0015283 \\
\hline GO:0007030 & Golgi organization ${ }^{\mathrm{D}}$ & 2 & $<0.0001$ & FBgn0261014, FBgn0033075 \\
\hline
\end{tabular}

${ }^{A} X_{a d j}^{2}$ refers to the significance of the Fisher's exact test with a Benjamini-Hochberg correction for multiple comparisons.

${ }^{B}$ Orthologues correspond to those pictured in Figure 3B (left panel) and represent Drosophila melanogaster genes that are listed in FlyBase.

${ }^{\mathrm{C}}$ Terms that were significant BP terms and were part of a significant gene annotation cluster.

DTerms that represented significant BP terms but did not form a significant gene annotation cluster.

bees. The data suggest that nutrients in pollen regulate the key developmental transitions that honey bees experience during early adult life as they prepare for critical brood care tasks.

Transcripts that were down-regulated in pollen deprived bees at both ages (i.e., due to the model main effect of age) included those with potential roles in development and muscle and motor function. These results agree with those of Ament et al. [19], who also found that the transcription of growth, development and muscle contraction genes was reduced in poorly fed nurses. It appears that poor diet is costly to the expression of genes involved in muscle function and growth from a rather early age. Whether this affects the ability of nurse bees to perform in-hive tasks or whether the lasting effects of starvation are evident in foragers remains to be studied. However, it is possible that traits such as foraging ability may be affected by poor diet in early adulthood.

Many more transcripts changed due to starvation in older bees (20,376 transcripts) compared to younger bees (173 transcripts). Of the transcripts where expression changed due to poor diet, there was more frequent down-regulation of gene expression in younger bees (78.5\%) compared to older bees (55.9\%). Transcriptional

Table 7 Biological processes up-regulated with age only in bees fed the rich diet

\begin{tabular}{lllll}
\hline BP term & Description & N & $\boldsymbol{X}_{\text {adj }}^{\mathbf{A}}$ & Orthologues $^{\mathbf{B}}$ \\
\hline GO:0055114 & Oxidation-reduction process & 2 & 0.0001 & FBgn0014032, FBgn0032945 \\
GO:0045087 & Innate immune response & 2 & $<0.0001$ & FBgn0030310, FBgn0043575 \\
GO:0006952 & Defense response & 2 & $<0.0001$ & FBgn0030310, FBgn0043575 \\
GO:0006955 & Immune response & 2 & $<0.0001$ & FBgn0030310, FBgn0043575 \\
GO:0009253 & Peptidoglycan catabolic process & 2 & $<0.0001$ & FBgn0030310, FBgn0043575 \\
\hline
\end{tabular}

${ }^{\mathrm{A}} \mathrm{Xdj}_{\mathrm{adj}}^{2}$ refers to the significance of the Fisher's exact test with a Benjamini-Hochberg correction for multiple comparisons.

${ }^{\mathrm{B}}$ Orthologues correspond to those pictured in Figure 3B (right panel) and represent Drosophila melanogaster genes that are listed in FlyBase. 


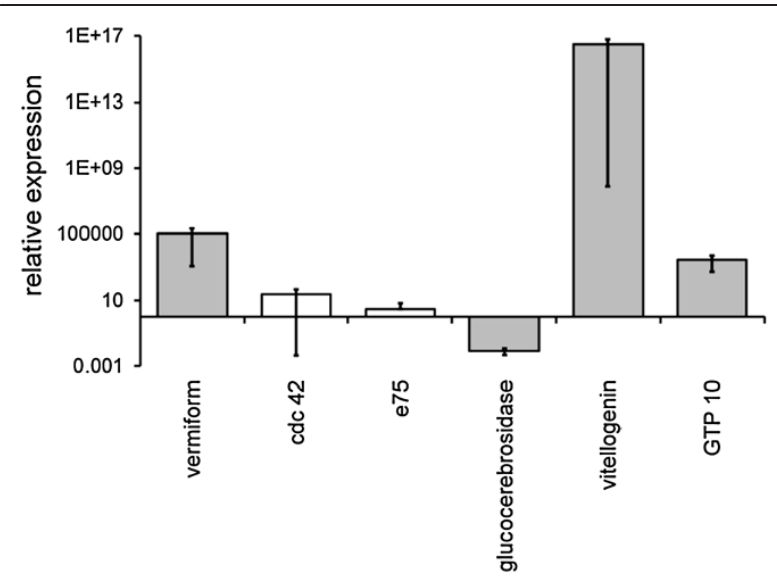

Figure 4 rt-PCR validation of RNA sequencing results. The

expression of genes in the fat bodies of bees fed a rich diet containing pollen relative to bees fed a poor diet that did not contain pollen is shown. Grey bars indicate significant over-expression $(>1)$ or underexpression ( $<1$ ) among $\mathrm{N}=3$ replicate samples using a Mann-Whitney test where the direction of expression agreed with predictions from the mRNA-seq experiment. Expression normalized relative to actin is shown and agreed with the results for expression normalized to GAPDH. White bars indicate results that, on average, agreed with the results of the mRNA-seq experiments but that were non-significant. Error bars represent the standard error of the mean relative expression value.

changes occurring due to starvation at $3 \mathrm{~d}$ were largely a subset of those occurring at $8 \mathrm{~d}$. Many more diet-related differences were observed only in bees deprived of pollen for $8 \mathrm{~d}$, but it is unclear whether the genes differentially expressed exclusively at $8 \mathrm{~d}$ are so due to the longer period of starvation or because specific processes that are turned on only at later ages are also impacted by diet. The expression of MRJPs, immune genes, and genes encoding detoxification enzymes declined due to poor diet exclusively at later ages. This group of downregulated transcripts included several members of the CYP4 and CYP6 cytochrome P450 families and one Delta GST, which have roles in insecticide detoxification [21], chitin-binding proteins that maintain the integrity of the peritrophic membrane, which plays a defensive role against chemicals and parasites [22], genes such as PGRP S3 and cytP450 4G11 whose down-regulation is associated with colony collapse disorder CCD; [23], and the trancripts dicer and argonaute that mediate immunity towards viruses that are sometimes prevalent in failing colonies [24,25]. One potential consequence, therefore, of pollen deprivation in nurses but not newly eclosed bees is reduced resistance to disease and insecticides.

Aging was associated with the overall decline of processes such as development, transcription, and mRNA splicing, and this decline was exacerbated in pollen deprived bees. Age-related transcriptional decline was more frequent in underfed bees (Tables 2 and Additional file 7: Table S5), and the processes occurring as well-fed bees aged were distinct from bees deprived of pollen (Figure 3). Bees fed a rich diet up-regulated genes involved in immunity and down-regulated those involved in tracheal system development and chitin metabolism, possibly as part of a developmental trajectory that prepares them for tasks outside of the hive. Poorly fed bees did not differentially regulate any of the age-related processes occurring in bees fed a normal diet. Pollen deprived bees showed down-regulation of genes involved in transcription and mRNA splicing and a non-coding RNA potentially involved in transcriptional regulation AncR-1; [26]. Loss of transcriptional regulation and the dysregulation of gene expression are associated with agerelated functional decline in a variety of taxa ([27-30]; but see [31]). The data therefore suggest that pollen deprivation may cause normal age-related transcriptional regulation to go awry, leading to various downstream effects. For example, aging bees deprived of pollen exhibited the reduced expression of genes involved in mushroom body and compound eye development. If such signals from the fat body impact brain signaling and development, this decrease could be a sign of diet-induced early foraging, as (1) early foraging is associated with nutrient restriction [6], (2) reduced volume of Kenyon cells in the mushroom body is associated with the transition to later age tasks such as foraging [32-34] and (3) the mushroom body is where sensory signals are integrated [35].

Further support for the hypothesis that aging is dysregulated in pollen-deprived bees becomes evident from comparing our results to examples presented in the literature, such as juvenile hormone esterase (JHE; which degrades juvenile hormone), vitellogenin, and hexamerin 70a. Nurses have highest levels of JHE in order to prevent the switch to foraging [36], as high levels of juvenile hormone are associated with the onset of foraging. We observed that for both fed and pollen starved bees JHE expression is higher in $8 \mathrm{~d}$ old bees compared to $3 \mathrm{~d}$ old bees. However, the magnitude of this increase was greater in starved bees and this increased expression with age was only significant in starved bees. Vitellogenin is very high in newly emerged workers, decreases slightly at days 1 and 2 post-eclosion, and then peaks at 3 days of age [37]. It then steadily decreases from this highest level until approximately 20 days of age $[38,39]$. In the present study, vitellogenin expression decreased as expected in well-fed bees aging from 3 days to 8 days old, but the difference was not significant. However, starved bees showed the opposite pattern, with expression increasing as they aged from $3 \mathrm{~d}$ to $8 \mathrm{~d}$, and the change in expression was significant. Cunha et al. [40] find that titres of the hexamerin $70 \mathrm{a}$ protein decrease a very small amount, if any between emergence and $7 d$ of age. We saw no difference in the expression of hexamerin 70a gene in old versus young bees when they were well-fed. However, when deprived of 
pollen, expression was significantly lowered as the bees aged. The aberrant expression of these genes in starved but not well-fed bees again suggests that aging is dysregulated in starved bees.

The expression of relatively few genes changed with respect to the main effect of age, yet there was measurable overlap in the patterns of expression when we compared the genes that were up- or down-regulated with age in the poor versus rich diets (for each diet analyzed separately; Figure 3). The transcripts that differed in expression due to the main effect of age showed similar patterns of significant up- or down-regulation for both diets and the magnitude of this change was the same for the different diets. Because we were interested in comparing the aging process in starved versus well-fed bees, we took the additional approach of determining what genes were differentially expressed due to age considering each diet separately. Figure 3 shows the overlap between diets in the transcripts that changed with age and this change was in the same direction for each of the diets. The main reason that these genes were not also significantly affected by the main effect of age was that the magnitude of the age-related change differed between diets, particularly in genes that were down-regulated as bees aged (Additional file 8: Figure S3). This difference in the magnitude of the age-related change in expression that is diet-dependent provides additional support for our hypothesis that the aging process itself differs due to diet.

Recent results from Ament et al. [19] and Alaux et al. [18] illustrate the utility of high-throughput analyses of the honey bee transcriptome for studying the benefits of good nutrition in honey bees. We can relate our results to those of these two studies by comparing the biological process $\mathrm{GO}$ terms differentially expressed in the present experiment, the terms found by Ament et al. [19], and the terms associated with the differentially expressed genes in Alaux et al. [18]. This post hoc analysis reveals a core group of biological processes that are up- or down-regulated in the abdomens of young pollendeprived A. mellifera. 60 biological process GO terms were up-regulated in pollen starved bees in all three studies (Figure 5), and included processes related to stress response, ovary development, and sensory development (Additional file 9: Table S6). 19 biological process GO terms were down-regulated in starved bees in all three studies (Figure 5), including those related to lipid metabolism and biosynthesis (Additional file 10: Table S7). These
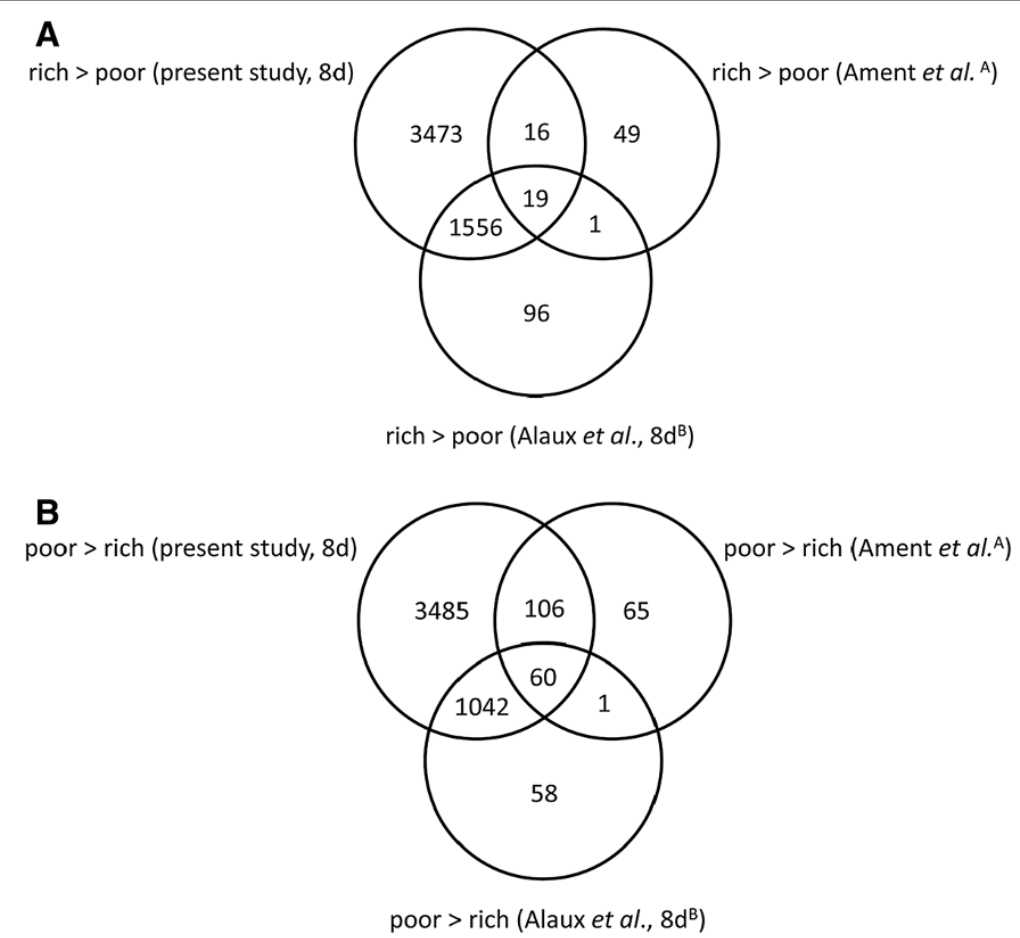

Figure 5 The core set of biological process impacted by diet. For transcripts that differed with diet, Venn Diagrams were constructed using the biological process gene ontology (GO) terms presented by Ament et al. [19], that were associated with the orthologues found in the present study for the 8d old bees only, and the BeeBase IDs presented by Alaux et al. [18]. Terms that were up-regulated in bees fed pollen are presented in panel $\mathbf{A}$. Terms that were up-regulated in starved bees are presented in panel $\mathbf{B}$. The superscript A indicates that diet-associated genes were identified in the abdominal carcasses for both nurses and foragers in Ament et al. [19], and the superscript B indicates that the entire abdomen (including the digestive tract) was utilized in Alaux et al. [18]. The data shown from the present study represent terms that were differentially expressed in abdominal carcasses of $8 \mathrm{~d}$ old bees. $3 \mathrm{~d}$ old bees were not included in the analysis. 
results suggest that in bees fed pollen, most of the gene activity in the abdominal carcass tissue of nurses is concentrated on energy production and storage. In contrast, starved nurses exhibit very different expression patterns, including the up-regulation of stress response pathways and, in the case of sensory development, pathways that are likely correlated with a transition to precocious foraging.

\section{Conclusion}

We found that substantial changes in gene expression occurred during early adult development and due to sub-optimal pollen intake in early adulthood. Dietinduced changes in gene transcription occurring in early age were largely a subset of those occurring at later age, but most signatures of starvation were only evident in older bees. The aging process itself differed when bees were deprived of pollen and the data are consistent with the hypothesis that poor diet causes normal agerelated development to go awry. The preponderance of evidence from this study and other recent studies $[18,19]$ indicates that adequate diet is critical to early worker development.

\section{Methods}

\section{Bees and experimental manipulations}

Three host colonies of Apis mellifera maintained for several months at the Carl Hayden Bee Research Center in Tucson, Arizona were used for dietary manipulations. The three host colonies were untreated (no miticides or antibiotics) 10 frame colonies with approximately 18,000 adults, 1 full frame face of brood, and 3.5 full frame faces of food stores. To collect newly emerged workers, sealed brood frames from approximately five colonies different from the host colonies used in the experiment were placed in a temperature-controlled room (approximately $34^{\circ} \mathrm{C}$ ) and all adults that emerged in an 18 hour period were collected. These callow bees ( $\leq 18$ hours of age) were marked using a small dot of non-toxic paint on their thorax and were added to the three host colonies, confined to each of two diets (a poor diet consisting of only stored honey or a rich diet of stored honey and bee bread) at a density of approximately one bee per $\mathrm{cm}^{2}$ ( 40-60 bees per cage) using wire cages pushed into drawn comb over either diet. Bees were confined for 3 or 8 days in July and August of 2011. To sum, each of the three host colonies contained two dietary treatments, and the bees were sampled after being confined to that treatment for either 3 or 8 days, yielding three replicate (host) colonies for each diet (poor versus rich) by age ( $3 \mathrm{~d}$ versus $8 \mathrm{~d}$ ) combination. Sampled bees were flash frozen in liquid nitrogen and maintained at $-80^{\circ} \mathrm{C}$ until further processing.

\section{Hypopharyngeal gland (HG) measurements}

Hypopharyngeal glands (HGs) of bees typically grow in preparation for nursing duties and this growth is impacted by the amount and source of dietary protein $[10,41]$. Our experimental design allowed trophallaxis (food sharing) with bees exterior to the treatment cage that had access to protein. To confirm that the bees caged over honey alone were indeed protein restricted compared to bees caged over the rich diet containing beebread and honey despite their access to other bees in the colony, the acini of HGs in bees aged $3 \mathrm{~d}$ or $8 \mathrm{~d}$ that were fed either diet were measured. HGs were dissected from approximately 5 bees per colony for each diet by age combination. 10 randomly selected acini per gland were visualized at $8 \mathrm{X}$ magnification. Acini area $\left(\mathrm{mm}^{2}\right)$ was measured using the Leica Applications Suite v.3.8.0 software. Measurements were analyzed using a mixed model repeated measures ANOVA testing for the effect of diet, colony, age, the two-way interactions between colony and diet, age and diet, age and colony, and the three-way interaction between age, colony, and diet. Each acinus was a repeated measure taken on the same bee and a compound symmetry covariance structure was employed to model the correlation among measures taken on the same bee. A post-hoc analysis was performed using the Tukey HSD test to determine whether there were significant differences in acini area between diets (rich versus poor diet) for each age (3d and $8 \mathrm{~d}$ old) and between ages for each diet.

\section{RNA extraction and library preparation}

Abdominal carcasses were dissected from three bees per colony per treatment by removing their head and wings before making a dorsal incision through their abdominal cuticle, exposing the abdominal organs and forming a cup structure where RNAlater (Life Technologies) was added. After removing the digestive tract, the remaining tissue (i.e., the fat body, reduced reproductive organs, exoskeleton, trachea, and muscles) was separated from the thorax and this abdominal carcass was preserved at $-80^{\circ} \mathrm{C}$ for subsequent RNA extraction. Three bees per host colony were pooled and total RNA was extracted using TriReagent (Invitrogen) according to the manufacturer's specifications. Total RNA integrity was confirmed using Agilent's 2100 Bioanalyzer.

Twelve mRNA sequencing (mRNA-Seq) libraries (2 ages $\times 2$ diets $\times 3$ replicate (host) colonies) were prepared using Illumina's TruSeq RNA Sample Preparation Kit according to the manufacturer's protocols. Briefly, poly-A containing mRNA was purified using poly-T oligoattached magnetic beads and was subsequently fragmented and primed for first-strand cDNA synthesis with random hexamer primers. After degrading the RNA template used for first-strand cDNA synthesis, synthesis of 
the second cDNA strand followed, yielding a doublestranded cDNA molecule. Adapters were ligated to both ends of the double-stranded cDNA molecules and adapter-ligated cDNAs were enriched using 15 cycles of PCR using adapter-specific primers. The libraries were validated according to the manufacturer's protocol and the approximately $350 \mathrm{bp}$ fragments were isolated from a 6\% Tris Base -Boric Acid-EDTA (TBE) PAGE gel and run through the Illumina sequencer for $2 \times 100$ cycles at a starting concentration of $12 \mathrm{pM}$ per library.

\section{Analysis of paired end sequence data}

Adaptor sequences were removed from each sequence and poor-quality reads were excluded using Trimmomatic [42] prior to the analysis of the $\sim 150$ base pair reads. The size distribution of the original transcripts for which sequence data was taken was first estimated by aligning the reads from each library separately to all publicly available $A$. mellifera RNA sequences listed in GenBank using the Burrows-Wheeler Aligner (BWA; [43]). Using the results from the BWA alignment, the expected mean and standard deviation of the inner distance between pairs was set for each library, and the reads were subsequently aligned to version 4.5 of the $A$. mellifera genome [44] with TopHat [45] version 1.4.0. The genome annotation contained NCBI reference sequence annotations and $a b$ initio predictions based on the A. mellifera version 4.5 genome [46]. First, we analyzed a full model to determine whether the effects of age, diet, and the interaction between age and diet significantly impacted gene expression using the edgeR package [47] and a Benjamini-Hochberg correction for multiple testing [48] at a 5\% false discovery rate. To test whether the effect of diet was different for $3 \mathrm{~d}$ old versus $8 \mathrm{~d}$ old bees, the effect of diet was investigated for each age separately. To test whether the effects of aging on gene expression differed with respect to diet, the effect of aging was studied separately for bees fed pollen and those that were not fed pollen.

Statistical analyses of differential expression yielded a corrected significance value for each exon that mapped to each mRNA transcript in the Apis mellifera version 4.5 genome annotation [44]. If $\geq 2$ exons mapped to the same transcript, we reasoned that the entire mRNA transcript (irrespective of isoform) was differentially expressed. Therefore, single exon exon transcripts (i.e., the only significantly differentially expressed exon mapping to an annotated transcript) were eliminated from further analyses. This approach was conservative because it eliminated the occurrence of false positives but came at a cost because it also removed single-exon transcripts from further analyses. In addition, we did not analyze alternative splicing events, which were beyond the scope of the study.
The significantly differentially expressed transcripts (containing $\geq 2$ exons) were subjected to further characterization. Drosophila melanogaster genes orthologous to the differentially expressed $A$. mellifera transcripts were identified by searching for reciprocal best BLAST hits between A. mellifera mRNA sequences and $D$. melanogaster proteins. A FlyBase gene ID (FBgn) [49] was assigned to all $A$. mellifera transcripts where a $D$. melanogaster orthologue was identified. GeneCoDis3 [50-52] was then employed to find enriched biological process gene ontology (BP GO) terms and gene annotation co-occurrence clusters (i.e., annotation clusters) that were significantly associated with the differentially expressed orthologues [50-52]. In addition to single BP GO annotations, annotation clusters represented significant relationships among biological process, molecular function, InterPro protein domain, and KEGG pathway annotations that were associated with the differentially expressed orthologoues [50-52]. A reference background list of all $D$. melanogaster genes orthologous to all A. mellifera RNAs was used to determine significant enrichment of BP GO terms and annotation clusters using a chi-square $\left(X^{2}\right)$ test corrected for multiple testing [48]. Significantly enriched BP GO terms that contained only one orthologue were discarded.

The annotated transcripts (containing $\geq 2$ exons) and orthologues that exhibited differential expression were compared. To address the hypothesis that diet and early adult development affect $A$. mellifera gene expression and physiology, annotated transcripts that increased or decreased due to starvation or as individuals aged from $3 \mathrm{~d}$ to $8 \mathrm{~d}$ old were compared and biological processes and annotation clusters corresponding to differentially expressed orthologues were analyzed for significant enrichment. To address the hypothesis that starvation affects younger and older workers differently, comparisons were made between annotated transcripts and orthologues that were affected by diet at either $3 \mathrm{~d}$ or $8 \mathrm{~d}$ of age. Venn Diagrams were constructed using the annotated transcripts that were differentially expressed due to diet at $3 \mathrm{~d}$ and those that were differentially expressed due to diet at $8 \mathrm{~d}$. To address the last hypothesis that development and aging are affected by diet, comparisons were made between annotated transcripts and orthologues that changed with age for bees fed either a rich diet or those fed a poor diet. Venn Diagrams were constructed using the annotated transcripts that were differentially expressed due to age for the rich diet and those that were differentially expressed due to age in bees deprived of pollen.

rt-PCR validation of gene expression changes due to diet To assess the predictive power of the mRNA-seq analyses, we measured the expression of six genes - vermiform (XM623720), cdc42 (XM_394608), E75 (NM_001080110), 
GTP-binding protein 10 (XM_396976), vitellogenin (NM_ 001011578), glucocerebrosidase transcript variant 1 (XM_ 393207; Additional file 11: Table S8) - that were significantly differentially expressed based on the previous mRNA-seq experiment. Fat bodies of $8 \mathrm{~d}$ old bees from three replicate host colonies $(N=3)$ were used. These bees were not used in the previous mRNA-seq experiment, but the colonies they came from shared similar population sizes, age distributions, and distribution of food stores to the three colonies used in the mRNA-seq experiments and to each other. Dietary manipulations, fat body dissections, and RNA isolations were as described above except RNA integrity was not assayed. To test that the dietary manipulations were successful, HGs were dissected and acini were measured in the manner described above. RNA was isolated from three pooled fat bodies per colony for each age and for each dietary treatment, yielding a total of six samples (3 host colonies x 2 diets). Isolated RNA was subjected to a DNase treatment (Ambion) and the lack of genomic DNA contamination was verified by performing a PCR on the RNA with A. mellifera actin (AB023025.1; Additional file 11: Table S8) and the following cycling conditions: 3 minutes at $94^{\circ} \mathrm{C}, 35$ cycles of 45 seconds at $94^{\circ} \mathrm{C}, 45$ seconds at $57^{\circ} \mathrm{C}$, and 45 seconds at $72^{\circ} \mathrm{C}$, and a final extension of $72^{\circ} \mathrm{C}$ for 10 minutes. cDNA was synthesized using the iScript cDNA synthesis kit (Bio-Rad) according to the manufacturer's protocol. The amplification efficiencies of all genes ranged between 95\% and 105\%. For each gene, a two-step qPCR was performed on the cDNA using SsoFast EvaGreen Supermix (Bio-Rad) according to the manufacturer's protocol and the following cycling conditions (annealing temperatures, $n$, are listed in Additional file 11: Table S8): 3 minutes at $95^{\circ} \mathrm{C}, 45$ cycles of 10 seconds at $95^{\circ} \mathrm{C}$ and 10 seconds at $n^{\circ} \mathrm{C}$, followed by a melt curve from $55^{\circ} \mathrm{C}$ to $95^{\circ} \mathrm{C}$ to confirm the lack of contamination and/or non-specific amplification. For each of the three colonies per diet, the threshold cycle $\left(C_{T}\right)$ values from three technical replicates were averaged for each target gene and two standards: $A$. mellifera actin (AB023025.1) and GAPDH (XM_393605; Additional file 11: Table S8). The average $C_{T}$ values of each target gene were normalized relative to the mean $C_{T}$ of each standard separately, accounting for primer amplification efficiencies [53]. Significant differences between the diets were determined using a Mann-Whitney/Wilcoxon test [54] and results were significant only if results were different from one for both of standards (actin and GAPDH).

\section{Availability of supporting data}

Additional file 6: Table S4 supporting the results of this article is available in the Dryad Digital Repository http:// doi.org/10.5061/dryad.pg2kb. All reads were submitted to the NCBI Sequence Read Archive under accession number SRA056350.

\section{Additional files}

Additional file 1: Table S1. Biological process gene ontology (GO) terms that showed higher expression in starved bees compared to bees fed pollen in both Ament et al. [19] and Alaux et al. [18].

Additional file 2: Table S2. Biological process gene ontology (GO) terms that showed reduced expression in starved bees compared to bees fed pollen in both Ament et al. [19] and Alaux et al. [18].

Additional file 3: Figure S1. The core set of biological process impacted by diet in previous studies. Biological process gene ontology (GO) terms that showed similar expression patterns in starved bees compared to bees fed pollen as determined by Ament et al. [19] and Alaux et al. [18].

Additional file 4: Figure S2. Hypopharyngeal gland $(H G)$ sizes of well-fed and nurse workers deprived of pollen, separated by host colony. $\mathrm{HG}$ size, as measured by $\mathrm{HG}$ acinus size $\left(\mathrm{mm}^{2}\right)$, is presented for bees raised for either 3 days ( $3 d$ ) or 8 days ( $8 d$ ) and fed a diet of honey alone $(H)$ or honey and pollen (HP) for colonies A, B, and C. Error bars represent standard error for the mean acinus size for the five individuals tested for each diet $x$ age combination. Significant results of a post-hoc Tukey-Kramer test on the mean acinus sizes for each diet by age combination are presented.

Additional file 5: Table S3. Summary of sequencing effort and read alignment to the Apis mellifera genome.

Additional file 6: Table S4. List of all differentially expressed transcripts. Data presented include (from left to right) the genome accession number (column A) and start and end positions (columns B and C) where that differentially expressed exon is located on the A. mellifera genome, the size of the exon (column D), the exon annotation in NCBI's GNOMON annotation (column E; the number after the colon indicates the exon number), the $D$. melanogaster orthologue matching the transcript (column F; FBgns indicate the FlyBase ID), the accession number and description for the mRNA at that genomic location (columns $\mathrm{G}$ and $\mathrm{H}$ ), and whether the full model yielded a significant result for the main effect of diet (column I) or age (column K) after a correction for multiple tests. Significance of pairwise contrasts between diets at 3 days (column M) and 8 days (column 0 ), and between young and old bees fed a poor diet (column Q) or a rich diet containing beebread (column S) are presented. Where the effect was significant, the direction and magnitude of the expression change between diets (column J), between ages (column L), between diets in only young bees (column N), between diets in older bees (column P), between ages in bees fed a poor diet (column R) and between ages in bees fed a rich diet (column T) are presented and were calculated using the expression estimates in columns $U$ through AF. Columns $U$ through AF include the fitted expression estimates for each exon and for each host colony $x$ age $x$ diet combination following a generalized linear model analysis of the raw expression values (edgeR; see text and $[47,55])$.

Additional file 7: Table S5. Exon-based analysis of the number of genes changing with respect to diet or age.

Additional file 8: Figure S3. Comparisons of the magnitude of age-associated (A) up- or (B) down-regulation in bees fed or deprived of pollen. For each exon that was significantly affected by age in bees fed a poor diet (deprived of pollen) or a rich diet (containing pollen), the average expression among host colonies was calculated for each age by diet combination. The magnitude of age-related change was then calculated by dividing the values obtained in young bees by the values obtained in old bees separately for each diet. This yielded estimates of up-regulation with age (panel A, values are greater than one because expression was higher in old bees compared to young bees) and downregulation with age (panel $B$, values are less than one because expression was higher in young bees compared to old bees) for bees fed each type of diet. Means of these values across exons are presented along with the standard error around this mean.

Additional file 9: Table S6. Biological process gene ontology (GO) terms that were up-regulated in starved bees compared to bees fed pollen in the present study, Ament et al. [19], and Alaux et al. [18]. 
Additional file 10: Table S7. Biological process gene ontology (GO) terms with reduced expression in starved bees compared to bees fed pollen in the present study, Ament et al. [19], and Alaux et al. [18].

Additional file 11: Table S8. List of rt-PCR primers used in this study.

\section{Competing interests}

The authors declare that they have no competing interests.

\section{Authors' contributions}

VC-H and KEA conceived of the experiments. VC-H designed the experiments. $\mathrm{VC}-\mathrm{H}, \mathrm{BMJ}, \mathrm{AW}$, and MRS performed the experiments. VC-H analyzed the data. KEA contributed reagents and materials. VC-H wrote the paper and all authors approved of the final manuscript.

\section{Acknowledgements}

The authors acknowledge Laurel Johnstone, Troy Comi, and Susan Miller for programming advice, the University of Arizona Genetics Core (Arizona Research Laboratories, Division of Biotechnology, http://uagc.arl.arizona.edu/), and the staff of the Carl Hayden Bee Research Center. We also thank Bruce Eckholm, Brendon Mott, Anna Himler, Kate Aronstein, Jay Evans and Gloria DeGrandi-Hoffman for helpful discussion and comments on previous versions of the manuscript. The ARS/USDA is an equal opportunity employer and provider.

\section{Author details}

${ }^{1}$ Carl Hayden Bee Research Center, USDA Agricultural Research Service, 2000 E. Allen Road, Tucson, Arizona 85719, USA. ${ }^{2}$ Department of Entomology, University of Arizona, Tucson, Arizona 85721, USA. ${ }^{3}$ Current address: Program in Ecology, Evolution, and Conservation Biology, University of Illinois at Urbana-Champaign, Urbana, IL 61801, USA. ${ }^{4}$ Current address: Department of Ecology, Evolution, and Organismal Biology, lowa State University, Ames, IA 50011, USA.

Received: 21 September 2013 Accepted: 4 February 2014 Published: 15 February 2014

\section{References}

1. Morse R, Calderone N: The value of honey bee pollination in the United States. Bee Culture 2000, 128(18):1-15

2. Winston ML: The Biology of the Honey Bee. Cambridge, Massachusetts: Harvard University Press; 1987.

3. Robinson GE: Regulation of division of labor in insect societies. Annu Rev Entomol 1992, 37:637-665.

4. Chan QW, Mutti NS, Foster L, Kocher SD, Amdam GV, Wolschin F: The worker honeybee fat body proteome is extensively remodeled preceding a major life-history transition. PLoS One 2011, 6(9):e24794.

5. Keller I, Fluri $P$, Imdorf A: Pollen nutrition and colony development in honey bees: part I. Bee World 2005, 86(1):3-10.

6. Toth AL, Kantarovich S, Meisel AF, Robinson GE: Nutritional status influences socially regulated foraging ontogeny in honey bees. J Exp Biol 2005, 208(Pt 24):4641-4649.

7. Page RE, Robinson GE, Britton DS, Fondrk MK: Genotypic variability for rates of behavioral development in worker honeybees (Apis mellifera L.). Behav Ecol 1992, 3(2):173-180.

8. Haydak $\mathrm{MH}$ : Brood rearing by honeybees confined to a pure carbohydrate diet. J Econ Entomol 1935, 28:657-660.

9. Haydak $\mathrm{MH}$ : The influence of pure carbohydrate diet on newly emerged honeybees. Ann Entomol Soc Am 1937, 30:252-262.

10. Crailsheim K: The protein balance of the honey bee worker. Apidologie 1990, 21:417-429.

11. Anderson KE, Sheehan TH, Eckholm BJ, Mott BM, DeGrandi-Hoffman G: An emerging paradigm of colony health: microbial balance of the honey bee and hive (Apis mellifera). Insect Soc 2011, 58(4):431-444.

12. Crailsheim K: Interadult feeding of jelly in honeybee (Apis mellifera L.) colonies. J Comp Physiol B: Biochem Syst Environ Physiol 1991, 161:55-60.

13. Standifer LN, Moeller FE, Kauffeld NM, Herbert EW Jr, Shimanuki H: Supplemental Feeding of Honey Bee Colonies. United States: Deparment of Agriculture; 1977.

14. DeGrandi-Hoffman G, Wardell G, Ahumada-Segura F, Rinderer T, Danka R, Pettis J: Comparisons of pollen substitute diets for honey bees: consumption rates by colonies and effects on brood and adult populations. J Apic Res 2008, 47:265-270.

15. Mattila HR, Otis GW: Influence of pollen diet in spring on development of honey bee (Hymenoptera: Apidae) colonies. J Econ Entomol 2006, 99(3):604-613.

16. van Engelsdorp D, Hayes J Jr, Underwood RM, Pettis J: A survey of honey bee colony losses in the U.S., fall 2007 to spring 2008. PLoS One 2008, 3(12):e4071.

17. van Engelsdorp D, Hayes J Jr, Underwood R, Pettis JS: A survey of honey bee colony losses in the United States, fall 2008 to spring 2009. J Apiculture Res 2010, 49(1):7-14.

18. Alaux C, Dantec C, Parrinello H, Le Conte $Y$ : Nutrigenomics in honey bees: digital gene expression analysis of pollen's nutritive effects on healthy and varroa-parasitized bees. BMC Genomics 2011, 12:496.

19. Ament SA, Chan QW, Wheeler MM, Nixon SE, Johnson SP, Rodriguez-Zas SL, Foster LJ, Robinson GE: Mechanisms of stable lipid loss in a social insect. J Exp Biol 2011, 214(Pt 22):3808-3821.

20. Corby-Harris V, Jones BM, Walton A, Schwan MR, Anderson KE: Transcriptional markers of sub-optimal nutrition in developing Apis mellifera nurse workers (Table S4. Table of differentially expressed genes). 2013. http://doi.org/ 10.5061/dryad.pg2kb.

21. Claudianos C, Ranson H, Johnson RM, Biswas S, Schuler MA, Berenbaum MR, Feyereisen R, Oakeshott JG: A deficit of detoxification enzymes: pesticide sensitivity and environmental response in the honeybee. Insect Mol Biol 2006, 15(5):615-636.

22. Merzendorfer $\mathrm{H}$, Zimoch L: Chitin metabolism in insects: structure, function and regulation of chitin synthases and chitinases. J Exp Biol 2003, 206(24):4393-4412.

23. Johnson RM, Evans JD, Robinson GE, Berenbaum MR: Changes in transcript abundance relating to colony collapse disorder in honey bees (Apis mellifera). Proc Natl Acad Sci USA 2009, 106(35):14970-14795.

24. Cornman RS, Tarpy DR, Chen Y, Jeffreys L, Lopez D, Pettis JS, van Engelsdorp D, Evans JD: Pathogen webs in collapsing honey bee colonies. PLoS One 2012, 7(8):e43562.

25. Cox-Foster DL, Conlan S, Holmes EC, Palacios G, Evans JD, Moran NA, Quan P-L, Briese T, Hornig M, Geiser DM, et al: A metagenomic survey of microbes in honey bee colony collapse disorder. Science 2007, 318(5848):283-287.

26. Sawata M, Takeuchi $H$, Kubo T: Identification and analysis of the minimal promoter activity of a novel noncoding nuclear RNA gene, AncR-1, from the honeybee (Apis mellifera L.). RNA 2004, 10(7):1047-1058.

27. Chambers SM, Shaw CA, Gatza C, Fisk CJ, Donehower LA, Goodell MA Aging hematopoietic stem cells decline in function and exhibit epigenetic dysregulation. PLOS Biol 2007, 5(8):e201.

28. Somel M, Khaitovich P, Bahn S, Pääbo S, Lachmann M: Gene expression becomes heterogeneous with age. Curr Biol 2006, 16(10):R359-R360.

29. Fraga MF, Ballestar E, Paz MF, Ropero S, Setien F, Ballestar ML, Heine-Suñer D, Cigudosa JC, Urioste M, Benitez J, et al: Epigenetic differences arise during the lifetime of monozygotic twins. Proc Natl Acad Sci USA 2005, 102(30):10604-10609.

30. Finch CE: Longevity, senescence, and the genome. Chicago: University of Chicago Press; 1994.

31. Pletcher SD, Macdonald SJ, Marguerie R, Certa U, Stearns SC, Goldstein DB, Partridge L: Genome-wide transcript profiles in aging and calorically restricted Drosophila melanogaster. Curr Biol 2002, 12(9):712-723.

32. Robinson GE: Regulation of honey bee age polyethism by juvenile hormone. Behav Ecol Sociobiol 1987, 20(5):329-338.

33. Withers GS, Fahrbach SE, Robinson GE: Selective neuroanatomical plasticity and division of labour in the honeybee. Nature 1993, 364(6434):238-240.

34. Withers GS, Fahrbach SE, Robinson GE: Effects of experience and juvenile hormone on the organization of the mushroom bodies of honey bees. J Neurobiol 1995, 26(1):130-144.

35. Erber J, Homberg U, Gronenberg W: Functional roles of the mushroom bodies in insects. In Arthropod brain: its evolution, development, structure, and functions. Edited by Gupta AP. New York: Wiley; 1987:588. xi.

36. Mackert A, Bitondi MM, Hartfelder K, Simoes ZL, do Nascimento AM: Identification of a juvenile hormone esterase-like gene in the honey bee, Apis mellifera L.-expression analysis and functional assays. Comp Biochem Physiol B Biochem Mol Biol 2008, 150(1):33-44.

37. Barchuk AR, Bitondi MM, Simoes ZL: Effects of juvenile hormone and ecdysone on the timing of vitellogenin appearance in hemolymph of queen and worker pupae of Apis mellifera. J Insect Sci 2002, 2:1. 
38. Piulachs MD, Guidugli KR, Barchuk AR, Cruz J, Simoes ZL, Belles X: The vitellogenin of the honey bee, Apis mellifera: structural analysis of the cDNA and expression studies. Insect Biochem Mol Biol 2003, 33(4):459-465.

39. Engels W: Reproduction and caste development in social bees. In Chemistry and Biology of Social Insects. Edited by Peperny VJ. Muchen; 1987:275-281.

40. Cunha AD, Nascimento AM, Guidugli KR, Simoes ZL, Bitondi MM: Molecular cloning and expression of a hexamerin cDNA from the honey bee, Apis mellifera. J Insect Physio/ 2005, 51(10):1135-1147.

41. Crailsheim K, Stolberg E: Influence of diet, age, and colony condition upon intestinal proteolytic activity and size of the hypopharyngeal glands in the honeybee (Apis mellifera L.). J Insect Physiol 1989, 35(8):595-602.

42. Lohse M, Bolger AM, Nagel A, Fernie AR, Lunn JE, Stitt M, Usadel B: RobiNA: a user-friendly, integrated software solution for RNA-Seq-based transcriptomics. Nucleic Acids Res 2012, 40(Web Server issue):W622-627.

43. Li H, Durbin R: Fast and accurate short read alignment with Burrows-Wheeler transform. Bioinformatics 2009, 25(14):1754-1760.

44. Honey Bee Genome Sequencing Consortium: Finding the missing honey bee genes: lessons learned from a genome upgrade. BMC Genomics. in review.

45. Trapnell C, Pachter L, Salzberg SL: TopHat: discovering splice junctions with RNA-Seq. Bioinformatics 2009, 25(9):1105-1111.

46. Kitts P: Genome assembly and annotation process. In The NCBI Handbook. Edited by McEntyre J, Ostell J. 2002.

47. McCarthy DJ, Chen Y, Smyth GK: Differential expression analysis of multifactor RNA-Seq experiments with respect to biological variation. Nucleic Acids Res 2012, 40(10):4288-4297.

48. Hochberg $Y$, Benjamini Y: More powerful procedures for multiple significance testing. Stat Med 1990, 9(7):811-818.

49. McQuilton P, St Pierre SE, Thurmond J: FlyBase 101-the basics of navigating FlyBase. Nucleic Acids Res 2012, 40(Database issue):D706-714

50. Carmona-Saez P, Chagoyen M, Tirado F, Carazo JM, Pascual-Montano A: GENECODIS: a web-based tool for finding significant concurrent annotations in gene lists. Genome Bio/ 2007, 8(1):R3.

51. Nogales-Cadenas R, Carmona-Saez P, Vazquez M, Vicente C, Yang X, Tirado F, Carazo JM, Pascual-Montano A: GeneCodis: interpreting gene lists through enrichment analysis and integration of diverse biological information. Nucleic Acids Res 2009, 37(Web Server issue):W317-322.

52. Tabas-Madrid D, Nogales-Cadenas R, Pascual-Montano A: GeneCodis3: a non-redundant and modular enrichment analysis tool for functional genomics. Nucleic Acids Res 2012, 40(Web Server issue):W478-483.

53. Pfaffl MW: A new mathematical model for relative quantification in real-time RT-PCR. Nucleic Acids Res 2001, 29(9):e45.

54. Yuan JS, Reed A, Chen F, Stewart CN Jr: Statistical analysis of real-time PCR data. BMC Bioinformatics 2006, 7:85

55. Robinson MD, McCarthy DJ, Smyth GK: edgeR: a bioconductor package for differential expression analysis of digital gene expression data. Bioinformatics 2010, 26(1):139-140.

doi:10.1186/1471-2164-15-134

Cite this article as: Corby-Harris et al.: Transcriptional markers of suboptimal nutrition in developing Apis mellifera nurse workers. BMC Genomics 2014 15:134.

\section{Submit your next manuscript to BioMed Central and take full advantage of:}

- Convenient online submission

- Thorough peer review

- No space constraints or color figure charges

- Immediate publication on acceptance

- Inclusion in PubMed, CAS, Scopus and Google Scholar

- Research which is freely available for redistribution

Submit your manuscript at www.biomedcentral.com/submit
C Biomed Central 